\title{
Comunicación

\section{Decisiones públicas sin diálogo público: análisis de los argumentos sobre el caso de la Vía Exprés vertidos en la prensa de Guadalajara1}

Public decisions without public dialogues:

Case study of the Via Express in Guadalajara's newspapers

\author{
RAÚL GERARDO ACOSTA GARCÍA, JUAN LARROSA-FUENTES Y \\ MARÍA MAGDALENA SOFÍA PALÁU CARDONA²
}

Este artículo presenta cómo se discutió la posible realización de una obra pública en Guadalajara. La obra, que no fue realizada, enfrentó a actores políticos y sociales. La investigación estudió cómo se construyó el diálogo público en cuatro diarios. A través del análisis de contenido de notas y opiniones publicadas y de los argumentos vertidos en ellas, los autores dan cuenta de la baja calidad del diálogo público.

PALABRAS CLAVE: Opinión pública, espacio público, déficit de diálogo público, análisis de contenido de prensa escrita.
This article analyzes the public debate regarding a proposed highway through the city of Guadalajara. This public work, which in the end was not carried out, was controversial and prompted heated debates between political and social actors. The research on which this paper is based scrutinized the coverage of the case in four local newspapers to evaluate the quality of public dialogue about the public work.

KEY WORDS: Public opinion, public space, lack of public dialogue, newspaper content analysis.

1 El análisis de contenido fue financiado por el Instituto Tecnológico y de Estudios Superiores de Occidente, y el análisis sobre diálogo público por una beca SEP-CONACyT de Investigación Básica.

2 Instituto Tecnológico y de Estudios Superiores de Occidente, México.

Correos electrónicos: rgacosta@iteso.mx; larrosa@iteso.mx; spalau@iteso.mx

Periférico Sur Manuel Gómez Morín 8585, C.P. 45604; Tlaquepaque, Jalisco, México. 
La forma en que el aparato gubernamental toma las decisiones para administrar la vida de millones de personas adolece, en México, de un considerable déficit de diálogos públicos. El presente estudio ofrece evidencias de las debilidades de la discusión pública en nuestro país, a través del seguimiento de un caso en la prensa de Guadalajara. ${ }^{3}$

En junio de 2010, el entonces gobernador del Estado de Jalisco, Emilio González Márquez, anunció un proyecto para la construcción de un viaducto de cuota llamado "Vía Exprés". Este viaducto estaba planeado para ser una vía elevada de alta velocidad, con $23 \mathrm{~km}$ de longitud. De acuerdo con la información difundida, la arteria permitiría que los automovilistas cruzaran Guadalajara, de oriente a poniente, de forma veloz y evitando los congestionamientos vehiculares. Esta iniciativa fue rechazada con vehemencia por organizaciones de la sociedad civil que llevaban años exigiendo una política integral de movilidad urbana que no privilegiara el automóvil. Durante tres meses, el tema de la "Vía Exprés" se posicionó como uno de los más importantes para la opinión pública jalisciense y tuvo un fuerte eco en la agenda mediática de la ciudad. Este artículo analiza la forma en que fue presentado el tema en los cuatro periódicos locales de mayor circulación en la ciudad y evalúa si existió voluntad, por parte de los involucrados, para establecer diálogos al respecto.

Lo que nuestros hallazgos exhiben es que no estamos acostumbrados, como sociedad, a promover diálogos incluyentes a partir de los cuales se tomen decisiones colectivas. Estudiar este hecho es crucial para comprender nuestra cultura política contemporánea y el funcionamiento institucional del Estado mexicano. El propósito que nos mueve al publicar este ensayo es un ánimo triple: analizar la forma en que se expone, en la esfera pública, un asunto de interés comunitario a cargo del gobierno estatal; examinar la labor periodística de diarios locales respecto de dicho tema, y ofrecer un análisis general sobre la cons-

3 Guadalajara es una de las tres ciudades más importantes del país por su desarrollo económico, territorio y población. Al momento de escribir este artículo (2012), la zona metropolitana de Guadalajara estaba integrada por ocho municipios que abarcan 2 mil $734 \mathrm{~km}^{2}$ y habitada por casi 4.5 millones de habitantes (Instituto Nacional de Estadística y Geografía [INEGI], 2012). 
trucción colectiva de decisiones cruciales en la vida comunitaria que este proceso representa. Un elemento central para este caso, y otros similares, es la aspiración a un sistema democrático que responda a los intereses de la opinión pública. Este anhelo forma parte del diseño institucional del aparato gubernamental y del discurso de los grupos de ciudadanos organizados que buscan aportar elementos para el debate.

El presente estudio busca responder a la siguiente pregunta: ¿es posible identificar una disposición por parte de los actores involucrados en el proyecto de la "Vía Exprés" para sostener diálogos públicos y así decidir su aprobación? Nuestra hipótesis es que no existe tal disposición y los resultados la confirman. Con estos objetivos en mente, el texto está dividido en tres partes. La primera incluye una explicación de los conceptos clave para comprender la situación (esfera pública, diálogo público, medios de comunicación) y la manera en que evaluamos la calidad de interacciones (calidad del diálogo); la segunda contiene el trabajo de investigación realizado, desde la metodología aplicada hasta un desglose de resultados; la tercera comprende el análisis de los datos y sus implicaciones, así como las conclusiones interpretativas que se desprenden del análisis.

\section{DIÁLOGOS PÚBLICOS Y DEMOCRACIA}

En una democracia liberal se espera que cualquier decisión que afectará a gran parte de la población se tome considerando las consecuencias a largo plazo y tras un periodo de estudio y discusión pública (Ackermann, 1999). Para ello existe un entramado de instituciones cuyo diseño estipula asegurar diversos análisis que ofrezcan claridad sobre las distintas implicaciones que puede traer la realización de una obra pública (políticas, económicas, ambientales, de salud, entre otras). La toma de decisiones que no se generan bajo las condiciones necesarias para considerar las distintas implicaciones de un proyecto refleja un desprecio por el diálogo. Además, se pone de manifiesto una falta de cuidado de los bienes públicos, que es una de las principales responsabilidades de las instituciones de gobierno. Para evitar este tipo de problemáticas, en muchos países y gobiernos locales se han instituido mecanismos para asegurar diálogos públicos, entendidos éstos como los procesos de- 
liberativos "sobre asuntos comunes a una sociedad" (Acosta, 2012b, p. 360). Estos mecanismos están expresados en organizaciones semigubernamentales en las que se ponen a debate diversos proyectos con especialistas independientes al aparato gubernamental o político.

En México sí existen dichos mecanismos, pero por lo general, son muy deficientes. Están, por ejemplo, los organismos públicos descentralizados, cuyos mandatos les exigen constituirse como mediadores entre los intereses de la sociedad y los del aparato de gobierno (Bautista, 2012). Además, varias instancias de gobierno, en el nivel local y federal, tienen representantes cuyas obligaciones son consultar a los afectados sobre planes y proyectos a realizar. Si los organismos u oficinas mencionados ejercieran sus mandatos, los diálogos públicos serían más productivos.

Independientemente de los procedimientos ideales, los diálogos públicos acontecen, con o sin fomento gubernamental, en la llamada esfera pública (Ruiz, 2009). Esta esfera no se trata de un espacio físico ni bien delimitado, sino de una serie de canales de comunicación donde todos los involucrados pueden aportar ideas, inquietudes o información. No significa que todos deban aportar algo, pero en la esfera pública se pueden identificar los temas que motivan o molestan a una comunidad. El concepto mismo de esfera pública se entiende solo cuando existe un mínimo anhelo democrático en la comunidad (Avritzer, 2002). En un sistema autoritario, la opinión de la población no importa (Bobbio, 1997).

En la explicación sobre el concepto de esfera pública que propuso en los años sesenta, Habermas (1989) aclara que como en otros usos del vocablo "público", no se trata de que toda la comunidad sea participativa todo el tiempo. El que un edificio sea público no significa que todos los habitantes de la zona vivan ahí. Se trata más bien de un inmueble que alberga a las instituciones del Estado, por lo que es "público", es decir, pertenece a la comunidad. Tampoco es equivalente a una declaración pública, pues ésta sería simplemente un pronunciamiento difundido ampliamente. La esfera pública es más bien un espacio virtual en el que se ponen de manifiesto las múltiples opiniones o posturas respecto de temas comunitarios. No implica en sí misma formas de solucionar las tensiones o fricciones que puedan existir entre 
dichas posturas, sino que se constituye como un espacio necesario para poder lograr negociaciones políticas que gocen de legitimidad social. Esto implica a su vez que existe una base democrática mínima en la vida colectiva a través de la cual se busque el bien común (Gutmann \& Thompson, 1996).

Sabemos que no existe ninguna comunidad política en la que los procesos de decisiones públicas sean satisfactorios para todos sus miembros. No consideramos a la democracia como un estado unívoco, sino como un proceso complejo en el que se establecen parámetros institucionales para una mejor administración de los bienes públicos y del bienestar social. A los países que han dejado atrás un régimen autoritario y buscan establecer un sistema de gobierno democrático se les suele llamar "en transición” (O’Donnell, Schmitter \& Whitehead, 1994). México forma parte de este grupo de naciones. Diversos estudiosos se han dedicado a analizar estos procesos particulares que llaman de "transición" o "consolidación” democrática (Cansino, 2000; Paoli, 2006; Valdés, 2006; Whitehead, 2002).

El principio trascendental del ideal democrático es que los gobiernos estén diseñados por y para los integrantes de la comunidad política en cuestión (Garretón \& Newman, 2003). Y precisamente de esto se tratan los diálogos públicos: de incluir la voz de los ciudadanos en debates colectivos para asegurar decisiones que beneficien a la comunidad y no solo a una minoría. Estos diálogos públicos se utilizan en las democracias consolidadas para considerar conocimientos especializados de profesionales independientes al gobierno, opiniones de los vecinos de obras proyectadas que serían afectados si se realizaran, y los puntos de vista de otros individuos o colectividades, como son las organizaciones de la sociedad civil (González \& Villar, 2003). Como herramientas para la toma de decisiones, aportan un alto grado de legitimación política, elemento fundamental para cualquier sistema de gobierno democrático. Es un ejemplo de lo que se ha dado por llamar "participación ciudadana" (Cunill, 1991; Forester, 1999). El concepto de bienestar colectivo a través de la participación ciudadana en la definición de políticas públicas se ha convertido en los últimos años en un elemento central de los sistemas democráticos. Como tal, está presente en el discurso oficial de los gobiernos estatal y municipal en Jalisco, exhibido en declaraciones 
de funcionarios y en documentos como el Plan Estatal de Desarrollo 2030 (Consejo Económico y Social de Jalisco [CESJAL], 2009). A pesar de esta insistencia discursiva, los mecanismos dedicados a facilitarla o administrar dicha participación adolecen de sesgos o censuras que han sido fuertemente criticados por grupos de la sociedad civil.

En todo este juego democrático, los medios de comunicación tienen un papel fundamental (Dennis \& Snyder, 1998; Sádaba \& Rodríguez, 2007). Es necesario, sin embargo, evitar mitos y estereotipos (que tienden a la idealización) en el análisis de los medios (Graber, 2003). El ejercicio periodístico permite a una comunidad conocer sus propias problemáticas, así como analizar y evaluar la labor de quienes ejercen el poder público. También permite un manejo de información que puede representar un riesgo para la democracia (Dahlgren, 2000). Como espacio virtual de comunicación comunitaria, los medios son la arena con mayor espacio de discusión y argumentación de la esfera pública:

Según Habermas, el ideal de esfera pública en cualquier sociedad descansaría, cuando menos, sobre dos pilares básicos: suficiente nivel de información de los ciudadanos y disposición para implicarse activamente en la discusión racional sobre los temas de interés colectivo. (Es así que podemos ubicar) el papel de los medios como espacios de información y debate y como espejo donde se mira la opinión pública para identificar los juicios prevalecientes a nivel social (Garcés, 2007, p. 58).

Los medios no son la única plataforma para el diálogo público; sin embargo, son un espacio privilegiado para que diversas voces sean visibles y se expresen sobre los temas de interés público (Thompson, 2011). Los periódicos, al dedicarse principalmente a ofrecer amplia información y análisis que concierne a una comunidad, son entonces espacios favorecidos para comprender las dinámicas de la opinión pública. El hecho de que la clase política haga referencia a la opinión pública para legitimar sus decisiones, muestra el peso que a su vez ésta tiene para la esfera política. Por lo mismo, los diarios son un medio clave para las instituciones de poder público, ya sea para cuestionarlas o apuntalarlas ante la sociedad. 


\section{EL CASO "VÍA EXPRÉS" Y LA METODOLOGÍA \\ DE ANÁLISIS DEL DIÁLOGO PÚBLICO}

Como parte de una estrategia para promocionar la realización de la "Vía Exprés", e influir en el ánimo de la opinión pública, el Gobierno del Estado de Jalisco presentó un video (Gobierno de Jalisco, 2010) acerca de los beneficios que se obtendrían con la construcción de esta obra. Semanas después de que apareció este mensaje, miembros de la organización civil "Ciudad para Todos", ${ }^{4}$ produjeron otro video (Carabes, 2010), en el que argumentaron en contra de la "Vía Exprés" y lo publicaron en Internet con la intención de presentar una visión crítica sobre el proyecto. El discurso y los argumentos vertidos en ambos videos se consideran, para efectos de este estudio, como los "discursos originales" de dos actores colectivos que podrían participar en un diálogo público en el que se discutieran las virtudes y defectos de construir una obra vial que afectaría a miles de ciudadanos en la zona metropolitana de Guadalajara.

El estudio que aquí presentamos analiza la cobertura del proyecto "Vía Exprés" en cuatro periódicos de Guadalajara entre junio y agosto de 2010. Los periódicos revisados fueron El Informador, PúblicoMilenio, Mural y La Jornada Jalisco (los diarios generalistas de mayor circulación en la ciudad).

Se analizaron 187 inserciones dedicadas al tema de la "Vía Exprés" que se clasificaron bajo dos categorías generales: informativas o de opinión. Las informativas corresponden a: nota informativa, fotografía, infografía y reportaje; las unidades cuyo propósito es la opinión son: columnas de opinión y correo del lector.

Este material recopilado lo analizamos a partir de tres procesos distintos, aportando con ello una metodología combinada. El primer

4 "Ciudad para Todos" es una organización ciudadana cuyos principios se manifiestan en "que la gente tenga el poder de decidir cómo se quiere mover y que, decida lo que decida, pueda hacerlo con seguridad, dignidad y rapidez; proteger las áreas verdes; recuperar espacios para la gente; que las opiniones de todos sean escuchadas; que el gobierno invierta de manera equitativa el erario público; que las personas con discapacidad sean capaces de trasladarse en su ciudad por sí solas; que las ciudades vuelvan a ser de los habitantes". 
trabajo corresponde al análisis del tratamiento general de la cobertura periodística, es decir, al análisis clásico de contenido (Krippendorff, 1990). El segundo aborda el desarrollo de la argumentación a favor y en contra del proyecto y su presentación en los diarios. El tercero atañe a la calidad del diálogo generado por los actores y vertido en la prensa. La segunda y tercera metodologías han sido desarrolladas por los propios investigadores participantes de este proyecto. A continuación se presentan los resultados de la investigación.

\section{EL TRATAMIENTO NOTICIOSO}

El diario que más publicó acerca del tema de la "Vía Exprés" fue Público-Milenio, que colocó en sus páginas 72 unidades noticiosas, mientras que La Jornada Jalisco es el que menos cobertura dio al tema al publicar 33 notas (18\% del total).

TABLA 1

COBERTURA EN LOS DIARIOS

\begin{tabular}{lcc}
\hline \multicolumn{1}{c}{ Periódico } & Número de notas & Porcentaje \\
\hline Público-Milenio & 72 & 38 \\
Mural & 45 & 24 \\
El Informador & 37 & 20 \\
La Jornada Jalisco & 33 & 18 \\
Total & 187 & 100 \\
\hline
\end{tabular}

Fuente: Elaboración propia.

El tema de la "Vía Exprés" estuvo presente en la agenda de los diarios locales durante 51 días, de los 84 que comprendió el periodo de observación (60\%). El proyecto de la "Vía Exprés" se presentó el 8 de junio de 2010 y la cobertura periodística le asignó relevancia únicamente las primeras dos semanas, en donde se concentra la mayor parte (108) de las 187 notas publicadas sobre el tema.

El periódico Público-Milenio es el que le dio una cobertura más constante en el tiempo, mientras que El Informador es el que menos atención le dio al caso de la "Vía Exprés" al tener varios días sin publi- 
car sobre este tema. La Tabla 2 muestra la cobertura sobre de la "Vía Exprés" por diario y por mes.

Con relación en el género periodístico con el que se presentó la información sobre la "Vía Exprés" encontramos 149 unidades del género informativo y 38 del género de opinión (tanto columnas como correos del lector); 68\% de las unidades del género de opinión fueron comunicaciones de los lectores. La mayor parte de las unidades de la categoría informativas fueron notas informativas y el reportaje es el género menos utilizado para abordar el tema. La frecuencia por tipo de unidad se presenta en la Tabla 3.

Como se puede ver, casi la mitad de las notas informativas estuvieron acompañadas por algún componente de información visual, ya sea fotografía del actor que hace una declaración o de infografías, en

TABLA 2

COBERTURA MENSUAL

\begin{tabular}{lcccrr}
\hline & $\begin{array}{c}\text { Público } \\
\text { Milenio }\end{array}$ & $\begin{array}{c}\text { El } \\
\text { Informador }\end{array}$ & $\begin{array}{c}\text { La Jornada } \\
\text { Jalisco }\end{array}$ & Mural & Total \\
\hline Junio 2010 & 38 & 27 & 21 & 30 & 116 \\
Julio 2010 & 14 & 5 & 10 & 9 & 38 \\
Agosto 2010 & 20 & 5 & 2 & 6 & 33 \\
Total & 72 & 37 & 33 & 45 & 187 \\
\hline
\end{tabular}

Fuente: Elaboración propia.

TABLA 3

GÉNEROS PERIODÍSTICOS UTILIZADOS

\begin{tabular}{lcc}
\hline \multicolumn{1}{c}{ Género } & Número de unidades & Porcentaje \\
\hline Nota informativa & 86 & 46 \\
Fotografía & 37 & 20 \\
Infografía & 14 & 8 \\
Reportaje & 12 & 6 \\
Opinión & 38 & 20 \\
Total & 187 & 100 \\
\hline
\end{tabular}

Fuente: Elaboración propia. 
su mayoría tomadas (copiadas) de la animación que se presentó en el video del proyecto del Gobierno del Estado de Jalisco. Es importante señalar que, aunque el género reportaje es el de menor frecuencia, es significativo que se hayan publicado 12 reportajes al respecto.

La relevancia que los diarios le dieron al tema de la "Vía Exprés" fue medida de acuerdo con la ubicación que los impresos le dieron en sus páginas. Se considera que un tema que aparece en la primera plana de un diario es más relevante que uno que se publica solo en interiores, así mismo si el tema aparece en una página impar es más relevante que si se ubica en una página par. Con estos parámetros observamos que $21 \%$ de las unidades analizadas salieron en primera plana, mientras que $79 \%$ solamente en interiores. Del mismo modo encontramos que $55 \%$ de las notas están en página impar, $42 \%$ en página par y $3 \%$ son notas que abarcan dos páginas consecutivas. Con estos datos se puede considerar que el tema tuvo una relevancia media, dado que solo 1 de cada 5 notas que abordan el tema mereció estar en la portada (primera plana) del diario. Esta afirmación se corrobora si revisamos la extensión de espacio que se asignó a estas unidades informativas (véase Tabla 4).

TABLA 4

ESPACIO ASIGNADO

\begin{tabular}{lcc}
\hline \multicolumn{1}{c}{ Tamaño } & Número de unidades & Porcentaje \\
\hline Dos planas & 2 & 1 \\
Una plana & 12 & 7 \\
Media plana & 40 & 21 \\
Cuarto de plana & 66 & 35 \\
Menos de un cuarto & 67 & 36 \\
Total & 187 & 100 \\
\hline
\end{tabular}

Fuente: Elaboración propia.

Según esta información, 71\% de las notas relativas a la "Vía Exprés" ocuparon un cuarto de plana o menos, en las páginas de los periódicos. Si revisamos esta misma información referida a cada uno de los diarios en particular, encontramos que únicamente el periódico Pú- 
blico-Milenio asignó una nota de dos planas al tema y El Informador y Público-Milenio concedieron una plana completa; el resto de las notas están en media plana o menos en Mural y La Jornada Jalisco.

Otra categoría de análisis del tratamiento noticioso es la revisión de la procedencia de las notas que se publican. En una primera mirada se revisa qué tipo de labor periodística se realiza, es decir, si la información la construyen los reporteros con su trabajo o proviene de agencias noticiosas o del correo del lector. En un segundo momento se analiza si la fuente de la información fue la declaración de alguno de los actores centrales o corresponde a la observación directa de un hecho, a una conferencia de prensa o simplemente a un boletín. Respecto de la procedencia de la información, encontramos que 51\% de las unidades analizadas son fruto de la labor de los reporteros, $22 \%$ proviene de agencias noticiosas, $14 \%$ de los lectores y $8 \%$ de la redacción. Al ver las fuentes o actos de los que surgen las notas (véase Tabla 5) encontramos que casi 1 de cada 2 notas es la declaración de los actores que participan del tema, que en su mayoría, ejercen el poder en la toma de decisiones y solo 1 de cada 5 unidades analizadas implican un papel activo por parte del reportero en la cobertura de la información haciendo observación o entrevistas.

TABLA 5

ACTO DEL QUE SE DESPRENDE LA NOTA

\begin{tabular}{lcc}
\hline \multicolumn{1}{c}{ Fuente } & Número de unidades & Porcentaje \\
\hline Declaración & 77 & 41 \\
Observación & 15 & 8 \\
Entrevista & 14 & 7 \\
Manifestación & 10 & 5 \\
Conferencia de prensa & 5 & 3 \\
Boletín de prensa & 2 & 1 \\
Encuesta & 1 & 1 \\
Lector & 26 & 14 \\
Otro & 37 & 20 \\
Total & 187 & 100 \\
\hline
\end{tabular}

Fuente: Elaboración propia. 
En términos de la cobertura noticiosa hay un dato más que resulta significativo para este análisis. De las unidades informativas, $93 \%$, es decir, 9 de cada 10 noticias publicadas, tienen como actor a un hombre, mientras que las mujeres solo aportan 1 de cada 10 notas, por lo que es evidente que tienen una participación menor en la discusión de este tema de interés público.

En un siguiente nivel de análisis del contenido de la prensa respecto del tema de la "Vía Exprés" revisamos qué actores tanto individuales como institucionales aparecen en las notas. En una clasificación básica encontramos que de las 429 menciones a actores concretos, 8 de cada 10 (343) hicieron referencia a actores gubernamentales, mientras que 2 de cada 10 (86) hicieron referencia a actores de la sociedad civil.

Respecto a las instituciones (véase Tabla 6) hay una clara tendencia a sobrerrepresentar al Poder Ejecutivo, mientras que el sector empresarial y los movimientos sociales son los de menor presencia en el tratamiento noticioso. El Poder Legislativo es el segundo actor institucional más mencionado, sin embargo, aparece cuatro veces menos que el Poder Ejecutivo. Estos datos parecen mostrar que la prensa no da cuenta, con suficiente énfasis, del trabajo y debate que, sobre el tema de la "Vía Exprés", se ha dado entre los diputados locales.

TABLA 6

INSTITUCIONES QUE APARECEN EN LAS NOTAS

\begin{tabular}{lcc}
\hline \multicolumn{1}{c}{ Institución } & Número de menciones & Porcentaje \\
\hline Poder Ejecutivo & 244 & 57 \\
Poder Legislativo & 63 & 15 \\
Organizaciones no gubernamentales & 38 & 9 \\
Universidades & 26 & 6 \\
Partidos políticos & 23 & 5 \\
Otros & 15 & 4 \\
Sector empresarial & 10 & 2 \\
Movimientos sociales & 10 & 2 \\
Total & 429 & 100 \\
\hline
\end{tabular}

Fuente: Elaboración propia. 
En cuanto a la mención de actores individuales encontramos 301 referencias a sujetos individuales. Los tres actores más mencionados fueron Emilio González Márquez, gobernador del Estado de Jalisco; Herbert Taylor Arthur, coordinador de Innovación y Desarrollo del Gobierno de Jalisco, y Raúl Vargas López, coordinador de la bancada del Partido de la Revolución Democrática (PRD) en el Congreso del Estado de Jalisco. Tanto Emilio González como Herbert Taylor impulsaron el proyecto de la "Vía Exprés" y obtuvieron 36\% de las menciones. Raúl Vargas se manifestó en contra de la construcción y representa $9 \%$ de las referencias a sujetos individuales en las notas analizadas. Entre los actores individuales de la sociedad civil que intervinieron en el debate sobre el proyecto de la "Vía Exprés" destacan Felipe Reyes y Jaime Meade, integrantes de la organización "Ciudad para Todos". A estos ciudadanos se les mencionó en cuatro ocasiones, es decir, menos de $2 \%$ del total, vertidas en las notas periodísticas. Por otro lado, hay 84 referencias a actores individuales que solo aparecieron una, dos o tres veces en las notas analizadas.

\section{LOS ARGUMENTOS}

\section{EN LOS TEXTOS PERIODÍSTICOS}

Como se señaló líneas arriba, se analizaron los argumentos originales presentados por el Gobierno del Estado de Jalisco y por las organizaciones de la sociedad civil a través de dos videos que circularon en Internet. De cada uno de estos videos se hizo una transcripción estenográfica y después se realizó una clasificación de los argumentos que aparecían en cada uno de los productos audiovisuales según ciertos temas (inversión, medio ambiente, calidad de vida, ahorro de tiempo, diseño urbano y flujo vehicular) y según su posición respecto de la construcción de la "Vía Exprés" (a favor o en contra). Para ello se conformó una matriz de argumentos, según su postura, de la realización de la obra pública (véase Tabla 7).

El ejercicio que realizamos fue rastrear estos argumentos originales, difundidos a través de ambos videos, en la cobertura informativa que los periódicos de Guadalajara hicieron sobre la "Vía Exprés". Es decir, los videos se convirtieron en una fuente de información y de opinión para argumentar a favor o en contra de la construcción de esta obra pública. A continuación se muestran nuestros hallazgos. 
TABLA 7

ARGUMENTOS ORIGINALES

\begin{tabular}{cll}
\hline Tema & \multicolumn{1}{c}{ Argumentos a favora } & \multicolumn{1}{c}{ Argumentos en contrab } \\
\hline Inversión & $\begin{array}{l}\text { La inversión la realiza la Ini- } \\
\text { ciativa Privada y no se utiliza- }\end{array}$ & $\begin{array}{l}\text { La obra pública debe ser realiza- } \\
\text { da por el gobierno. }\end{array}$ \\
& & Es necesario usar recursos públi- \\
& & cos en alternativas de movilidad \\
& & como ciclovías, sistema de trans- \\
& & porte público, entre otras.
\end{tabular}

Ambiental Al facilitar trayectos cortos y ágiles, se reducen las emisiones contaminantes.

Los automovilistas realizarán

Calidad de vida el trayecto en menos tiempo y les permitirá pasar más tiempo para compartir en familia. Es una vía segura.

La obra generará mayor convivencia entre habitantes.

La vía se congestionará, habrá más autos y por lo tanto aumentarán las emisiones contaminantes.

La obra partirá la ciudad en dos y una de las partes quedará más afectada, convirtiéndose en un gueto.

La parte baja de los puentes de esa zona será peligrosa y sucia.

Los vecinos que habiten cerca de la vía rápida realizarán menos ejercicio y estarán expuestos a la contaminación.

Ahorro Es una vía rápida que transEste tipo de vías se congestionan de tiempo portará a las personas en menos tiempo. rápidamente, por lo que no habrá ahorro de tiempo.

La velocidad continua será de $90 \mathrm{~km} / \mathrm{h}$.

Esta obra vial contará con sistemas inteligentes de peaje.

a Los argumentos a favor fueron presentados en el video realizado por el Gobierno del Estado de Jalisco.

b Los argumentos en contra fueron presentados en el video realizado por la organización civil "Ciudad para Todos". 


\begin{tabular}{|c|c|c|}
\hline Tema & Argumentos a favora & Argumentos en contrab \\
\hline $\begin{array}{l}\text { Diseño } \\
\text { urbano }\end{array}$ & $\begin{array}{l}\text { Soluciones similares se han } \\
\text { implementado en grandes ciu- } \\
\text { dades como Los Ángeles, To- } \\
\text { kio o Seúl. } \\
\text { La obra se ha diseñado con los } \\
\text { más altos estándares en el ni- } \\
\text { vel mundial. }\end{array}$ & $\begin{array}{l}\text { La obra propuesta es una solu- } \\
\text { ción de } 1950 \text { para un problema } \\
\text { del siglo XXI. Hay experiencia } \\
\text { en muchas ciudades en donde no } \\
\text { se resolvió el problema. } \\
\text { Con una obra de este tipo, la ciu- } \\
\text { dad se volverá más disfuncional. }\end{array}$ \\
\hline $\begin{array}{l}\text { Flujo } \\
\text { vehicular }\end{array}$ & $\begin{array}{l}\text { Esta obra disminuirá la con- } \\
\text { gestión vehicular. }\end{array}$ & $\begin{array}{l}\text { Esta vía aumentará el número de } \\
\text { autos en circulación, pues está } \\
\text { visto que las nuevas vías generan } \\
\text { tráfico nuevo. }\end{array}$ \\
\hline
\end{tabular}

Fuente: Elaboración propia.

En 128 de las 187 unidades periodísticas analizadas aparece algún tipo de argumentación. En 56 casos las notas mostraron argumentos a favor de la obra y en 72 se expusieron argumentos en contra. La Tabla 8 muestra la distribución de notas por periódico en relación con los argumentos que se exponen.

La tabla demuestra que los periódicos Mural y El Informador presentaron una cobertura equilibrada, pues publicaron casi el mismo número de notas a favor y en contra de la obra. Los diarios PúblicoMilenio y La Jornada Jalisco publicaron el mayor número de notas con argumentación crítica a la construcción de la "Vía Exprés".

TABLA 8

ARGUMENTOS EN NOTAS PERIODÍSTICAS

\begin{tabular}{lccc}
\hline \multicolumn{1}{c}{ Periódico } & $\begin{array}{c}\text { Número de notas } \\
\text { a favor }\end{array}$ & $\begin{array}{c}\text { Número de notas } \\
\text { en contra }\end{array}$ & Total \\
\hline Público-Milenio & 16 & 25 & 41 \\
Mural & 15 & 17 & 32 \\
La Jornada Jalisco & 10 & 15 & 25 \\
El Informador & 15 & 15 & 30 \\
Total & 56 & 72 & 128 \\
\hline
\end{tabular}

Fuente: Elaboración propia. 
Los argumentos favorables a la realización de la obra estuvieron relacionados con los temas de "inversión" y "flujo vehicular". En las piezas informativas, las voces predominantes fueron las del gobernador del Estado de Jalisco, Emilio González Márquez, del presidente municipal de Tlaquepaque, Miguel Castro Reynoso y del secretario general del Estado de Jalisco, Fernando Guzmán Pérez Peláez. Estos argumentos se refieren al beneficio que implica que la inversión se realice desde la iniciativa privada y a la disminución de la congestión vial.

Los argumentos contra la construcción de la obra que aparecen con frecuencia estuvieron relacionados con los temas "calidad de vida", "inversión" y "diseño urbano". Estos argumentos fueron expuestos a través de las declaraciones de Raúl Vargas López, coordinador de la bancada del PRD en el Estado de Jalisco; Olga Gómez Flores, diputada estatal por el PRD y Alejandro Mendo Gutiérrez, investigador del Instituto Tecnológico y de Estudios Superiores de Occidente (ITESO). Estos argumentos aludieron a los perjuicios que tendría partir la ciudad en dos y al peligro que la parte baja de los puentes representa para los habitantes de la zona. Adicionalmente refieren que el problema de movilidad urbana debe considerar el uso de recursos públicos en verdaderas alternativas de movilidad y al hecho de que la ciudad se vuelve disfuncional con este tipo de vías rápidas.

Es importante señalar que todos los temas (véase Tabla 7) de los videos originales aparecieron en la cobertura informativa de los diarios. Sin embargo, hubo temas que se presentaron con poca frecuencia como lo son "calidad de vida" o "ambiental".

\section{LA CALIDAD DEL DIÁLOGO PÚBLICO}

Hasta aquí es posible hablar de la existencia de suficiente información tanto a favor como en contra que muestran el abanico de opiniones y datos presentes en la sociedad tapatía. Sin embargo, como esperamos que demuestre esta parte de nuestro texto, tal información pierde su valor si no forma parte de un proceso dialógico en el que exista una disposición a incorporar críticas para evaluar pros y contras. Esto es, si no forma parte de un diálogo público. En este sentido, utilizamos el concepto de "calidad del diálogo público" (Acosta, 2012a), debido a 
que nos ayuda a determinar no solo si existen los elementos discursivos de un diálogo, sino también si estos se presentan de forma que faciliten un proceso de deliberación colectiva.

La "Vía Exprés" fue un proyecto de infraestructura urbana que, de haber procedido, habría afectado la vida de muchas personas. Como con cualquier otro proyecto, habría beneficiarios y afectados. Este tema en particular cobró relevancia local al ser criticado ampliamente por grupos de la sociedad civil al poco tiempo de haber sido presentado. El hecho de que las instituciones que aparecen en las notas son mayoritariamente gubernamentales (véase Tabla 6), habla de una cobertura periodística en la que la voz del gobierno tiene una sobrerrepresentación.

En este contexto, presentamos las categorías de análisis que utilizamos para evaluar la calidad de los diálogos públicos. Estas categorías se han desarrollado para evaluar la calidad de los diálogos públicos y son el resultado de un proceso doble de investigación y análisis colegiado de los resultados. En total, son cinco grados que van del mínimo posible, cuando el diálogo es inexistente, hasta el máximo, definido como ideal. Entre ambos extremos están: los diálogos simulados, que sirven a quien detenta una posición de poder para legitimarse; los desordenados, que aportan algunas señales de lo posible a través de mejores diálogos, pero cuyo desorden no permite un intercambio ideal entre los participantes, y los prósperos, que permiten a los copartícipes una experiencia plena de diálogo.

Las referencias a calidad responden a tres esferas de significación: en el procedimiento, en los contenidos y en el resultado (Morlino, 2005). Esta combinación de formas de comprender la calidad ayuda a relacionar las expectativas con la acción, sus elementos y sus resultados. Las categorías que se presentan a continuación fueron definidas como parte de un análisis comparativo de diversas situaciones. El resultado corresponde a un nivel de abstracción de los mecanismos que fueron identificados como necesarios de acuerdo con los sujetos de investigación involucrados. Estas categorías, que hemos denominado como "factores", se construyeron a partir de distintos procesos de investigación empírica (Acosta, 2012a). 
Debido a que cada uno de los factores para evaluar la calidad del diálogo implica un nivel de complejidad respecto de procesos de significación y práctica social, fue elaborada una matriz con gradación de cumplimiento de cada uno de los factores (véase Tabla 9). Esto a su vez permite evaluar la calidad de cualquier diálogo a partir de la característica dominante de cada factor durante el mismo. La razón principal para definir la calidad de esta manera es que en ningún caso puede decirse que cada factor existe o no existe, sino que se presenta de distinta manera.

TABLA 9

MATRIZ CON GRADACIÓN DE LOS FACTORES PARA EVALUAR LA CALIDAD DEL DIÁLOGO

\begin{tabular}{|c|c|c|c|c|c|}
\hline \multirow[t]{2}{*}{ Factor } & \multicolumn{5}{|c|}{ Tipos de diálogo } \\
\hline & Inexistentes & Simulados & $\begin{array}{c}\text { Desorde- } \\
\text { nados }\end{array}$ & Prósperos & Ideales \\
\hline Legitimidad & Imposición & Parcialidad & Renuencia & Confianza & $\begin{array}{l}\text { Reconoci- } \\
\text { miento }\end{array}$ \\
\hline Socialización & Opacidad & $\begin{array}{l}\text { Ambigüe- } \\
\text { dad }\end{array}$ & Sesgo & Conveniencia & $\begin{array}{l}\text { Transpa- } \\
\text { rencia }\end{array}$ \\
\hline Visión común & $\begin{array}{l}\text { Indiferen- } \\
\text { cia }\end{array}$ & Engaño & $\begin{array}{l}\text { Inconsis- } \\
\text { tencia }\end{array}$ & Interés & Estrategia \\
\hline $\begin{array}{l}\text { Código } \\
\text { común }\end{array}$ & Ausencia & $\begin{array}{l}\text { Desaten- } \\
\text { ción }\end{array}$ & $\begin{array}{l}\text { Elementos } \\
\text { básicos }\end{array}$ & Acuerdo & $\begin{array}{l}\text { Conven- } \\
\text { ción }\end{array}$ \\
\hline Evaluación & Desprecio & Prejuicio & Inequidad & $\begin{array}{l}\text { Semi } \\
\text { estructura }\end{array}$ & Rigor \\
\hline
\end{tabular}

Fuente: Acosta (2012a, p. 18).

Según los factores expuestos en la Tabla 9, para evaluar la calidad del diálogo en el caso de la "Vía Exprés", encontramos que los de "socialización" y "evaluación" colocan a este diálogo como inexistente; los factores "legitimidad" y "visión común" ubican al proceso como un diálogo simulado y el de "código común" lo ubica como un diálogo desordenado.

Detallando lo anterior encontramos que el factor "legitimidad" hace referencia al reconocimiento mutuo entre los actores. En el caso de la 
"Vía Exprés" los medios de comunicación visibilizan un diálogo que se califica de "parcial", es decir, el actor dominante, en este caso el Poder Ejecutivo, aparece como un actor con superioridad respecto de los actores que representan a las organizaciones ciudadanas e incluso al Poder Legislativo estatal. Líneas arriba encontramos que el Poder Ejecutivo aparece en $57 \%$ de las unidades informativas mientras que los movimientos sociales, organizaciones no gubernamentales y universidades aparecen en 17\% de las notas. Es decir, hay parcialidad en la exposición de los argumentos a favor de la obra por encima de los argumentos en contra de la misma.

El factor "socialización" se refiere a la circulación de la información básica sobre el asunto central del diálogo, se reconoce cuando se hace referencia a estudios realizados o datos relevantes respecto del asunto central. En el caso de la "Vía Exprés" la información sobre estudios técnicos y de impacto social y ambiental no se socializaron abiertamente entre los actores que intervienen en el diálogo. Esto queda claro en algunas notas informativas como las siguientes:

Un Gobierno que se niega a discutir nada con sus ciudadanos, que no hace paneles con sus expertos, que no responde las quejas vía Twitter o correo electrónico y que dice tener un plan sustentable de movilidad que no le ha mostrado nunca a nadie. Por cierto ... quiénes son los expertos que el Gobierno dice tener, dónde están los estudios, por qué no se comenta nada ni se discute nada con la ciudadanía (Zul, 2010); "Los diputados locales han manifestado al Gobierno de Jalisco estar a la espera de más información sobre la Vía Express, para poder autorizar la entrega de la concesión del viaducto elevado, afirman que los datos que entregó el Ejecutivo son insuficientes." ("Emilio evita polémica por Vía Express", 2010).

Es por ello que desde el factor de socialización se puede clasificar este diálogo como inexistente, por la opacidad en la circulación de información respecto de los estudios técnicos y de impacto ambiental que no fueron expuestos ni a la ciudadanía ni a los diputados locales.

En términos del factor "visión común" el diálogo se podría clasificar como un diálogo simulado en el que no hay claridad entre todos los actores sobre el propósito del diálogo. Existe coincidencia en lo que origina 
el diálogo pero no en las metas. Cada uno de los actores intenta convencer a los otros de un fin común cuando en realidad lo que les interesa es un fin particular. En el caso de la "Vía Exprés" las notas periodísticas dan cuenta de las posturas opuestas de diversos actores: autoridades del Poder Ejecutivo estatal y de algunos municipios además de diputados de la bancada panista, que se manifiestan a favor de la obra y exponen los beneficios; por otro lado, miembros de la organización "Ciudad para Todos" o investigadores de la Universidad de Guadalajara (UdeG) que plantean los perjuicios que traería la obra. En los discursos recogidos por la prensa local no hay coincidencias respecto de la meta a alcanzar en este tema, parecen dos proyectos de movilidad urbana en pugna.

Con relación en el factor "visión común" que se define como la manera en que un grupo de individuos puede comunicarse pasa, necesariamente, por un código compartido. Este puede ser un código, una serie de referentes culturales específicos o una jerga especializada. El código que comparten los actores que intervienen en este diálogo parece ser el de los "elementos básicos": hay algunos elementos básicos compartidos que podrían ser utilizados a manera de un código común, pero son más fortuitos que logrados por acuerdos entre las partes. Es así que todos los actores manifiestan la necesidad de un proyecto de movilidad urbana integral para Guadalajara, proponen una decisión democrática y transparencia en el manejo de los recursos; sin embargo, estos parecen ser elementos discursivos que no tienen el mismo significado o no llevan a las mismas concreciones en la visión de los actores. El diálogo así visto podría clasificarse como "desordenado", es decir, con elementos de visión común pero sin llegar a compartir estos referentes y sus posibles concreciones.

En relación con el factor "evaluación" que se define como la disposición de todos los involucrados por valorar la manera en que se ha realizado un diálogo, permite a su vez compartir los aprendizajes y asimilar los errores cometidos para mejorar la posibilidad de una discusión futura, en el caso de la "Vía Exprés" queda caracterizado como "inexistente" dado que hay un elemento de "desprecio", es decir, no hay ánimo entre las partes involucradas para evaluar el diálogo. Esto aparece en el discurso de algunos de los actores que se recoge en las unidades informativas analizadas, es el caso del gobernador quien afirma: 
Estamos en la propuesta de las cosas que pueden ayudar, a nosotros nos toca proponer resultados, otros que los obstaculicen, a nosotros nos toca ponernos del lado de la gente para ayudarles a que nos podamos mover todos con agilidad, otros son los que buscan el cómo detener los proyectos (Mendoza, 2010).

Afirmaciones que tienen un tono descalificador de quienes son interlocutores y que marcan un sentido de desprecio por los que argumentan en contra de la obra.

\section{CONCLUSIONES: EL CASO DE LA "VÍA EXPRÉS"}

COMO MUESTRA DE LOS DÉFICITS EN EL DIÁLOGO PÚBLICO

El caso de la "Vía Exprés" ha sido muy significativo para una buena parte de la opinión pública de Jalisco, pues se convirtió en uno de los episodios en los que se hizo evidente un desencuentro entre las ideas de desarrollo urbano del Gobierno del Estado y amplias franjas de la población. Para el equipo que presenta este trabajo de investigación académica, el caso de la "Vía Exprés" resultó ser pertinente para su estudio porque representa un ejemplo de cómo es que se desarrollan los diálogos públicos en la ciudad de Guadalajara. Evidentemente existen muchos temas que podrían haber sido estudiados, sin embargo éste nos pareció paradigmático porque la "Vía Exprés" fue, a la postre, uno de los proyectos políticos más importantes del Gobierno del Estado y porque logró aglutinar a una serie de movimientos sociales y organizaciones civiles que anteriormente no tenían contacto entre sí, o que incluso eran antagónicas. El tema de la obra pública es importante para cualquier gobierno, pues es una parte de su trabajo que adquiere una gran visibilidad ante la ciudadanía, y en el caso de la "Vía Exprés" no fue la excepción.

Como se explicó detalladamente en las páginas anteriores, el objetivo de esta investigación fue observar el desarrollo de un diálogo público en los periódicos de Guadalajara. Decidimos centrar nuestra atención en los diarios porque estos conforman una parte fundamental, aunque no exclusiva, del espacio público de esta ciudad. El mayor reto de este trabajo fue construir los instrumentos de investigación para poder observar y medir este diálogo público, pues en la literatura especia- 
lizada no encontramos un ejercicio como el que aquí se presenta. Con estas ideas en mente, decidimos observar durante tres meses (tiempo que duró el conflicto en la agenda mediática), todos los ejemplares de los cuatro periódicos más influyentes de la ciudad, para conocer las características del diálogo público. Una de nuestras premisas de inicio fue que los distintos dialogantes tendrían que recurrir a una fuente primaria de opinión e información para construir sus argumentos en torno al caso de la "Vía Exprés"; por ello establecimos dos fuentes primarias de información, a las que llamamos "discursos originales" y lo que nos interesaba observar era cómo es que éstos se colaban en la información publicada en los diarios. Los resultados, que ya hemos presentado, demuestran que estos discursos originales sí fueron parte fundamental de la discusión pública que se suscitó en torno a este tema.

Una vez que el Gobierno del Estado de Jalisco estableció el tema de la "Vía Exprés" en la agenda pública, diversos medios y organizaciones sociales comenzaron a debatir al respecto. El seguimiento de los "discursos originales" en la agenda de los medios de comunicación permitió observar cómo ciertos actores sociales y políticos fueron construyendo (o copiando), sus propios discursos a favor o en contra de la construcción de la "Vía Exprés", pero también cómo los medios fueron presentando el tema ante la opinión pública.

La conclusión de este trabajo de investigación es que en Guadalajara sí existen diálogos públicos pero de mala calidad. Es muy importante hacer notar que las discusiones públicas en torno a la movilidad urbana o el medio ambiente han ido en aumento en los últimos años, lo que da cuenta de una esfera pública en movimiento y en desarrollo. Sin embargo, al revisar la historia política y cultural de la ciudad es claro que durante décadas, y bajo regímenes monolíticos y en algunos casos, autoritarios, el diálogo público sobre estos temas era prácticamente inexistente. El tema de la "Vía Exprés" muestra cómo es que una ciudad comienza a discutirse a sí misma, pero con muchas limitaciones y pocas herramientas que posibiliten un diálogo público de calidad.

En este estudio de caso encontramos cuatro elementos que limitan el diálogo público en la Zona metropolitana de Guadalajara: un gobierno que no abre espacios de comunicación con la sociedad civil, y que por el contrario, buscar imponer sus políticas públicas de forma unilateral; 
un sistema de medios de comunicación, en este caso de periódicos, que hace poco periodismo de investigación, que privilegia la voz de los funcionarios públicos y relega la participación de otros actores sociales; una amplia participación de ciudadanos que escriben "quejas" en la sección de correo de lector de los diarios, pero que no trascienden a esta acción (no se organizan ni se integran a asociaciones o movimientos sociales), y una sociedad civil (organizaciones y movimientos) muy activa, pero con estrategias poco efectivas para integrase al diálogo público.

La evidencia empírica que presentamos en este trabajo corrobora las afirmaciones anteriores. Es muy significativo, por ejemplo, que $41 \%$ de los trabajos periodísticos estudiados tengan como fuente informativa principal una "declaración" y no un trabajo de investigación mucho más complejo o que el Poder Ejecutivo de Jalisco acapare las menciones en las notas informativas, fotografías e infografías de los periódicos, apareciendo en $57 \%$ de las ocasiones; también es muy elocuente el hecho de que los dos funcionarios públicos promotores de este proyecto hayan tenido la más alta presencia de todo el corpus informativo $(36 \%)$. Estas tendencias configuran un periodismo que privilegia las declaraciones de funcionarios públicos para la construcción de una nota informativa y que deja a un costado otras posibilidades de investigación periodística como la entrevista, la observación, la obtención de datos a través del sistema público de información, el análisis hemerográfico y de documentos, la consulta a especialistas, entre otras. Por otro lado, se corrobora una alta dependencia del periodismo mexicano a las "fuentes oficiales", es decir, al predominio de funcionarios públicos en detrimento de la aparición de otros actores sociales. Esta tendencia, que ya ha sido corroborada en otros estudios, da cuenta de un periodismo "oficialista".

Por otro lado, es evidente que el tema de la "Vía Exprés" fue muy importante para la sociedad, pues los espacios en los cuales los lectores pudieron participar se vieron saturados de correos en los que criticaban o apoyaban esta obra pública. Los lectores, incluso, abordaron este tema en más ocasiones que los analistas y columnistas de los diarios: de cada diez notas de opinión que registramos, seis fueron cartas de lectores. Además, los periódicos reportaron una amplia movilización de organizaciones civiles y movimientos sociales críticos al proyecto. Sin 
embargo, ni los lectores ni la sociedad civil organizada crearon estrategias lo suficientemente poderosas para equilibrar el diálogo público.

Los tres rasgos del diálogo público en Guadalajara aquí expuestos (periodismo oficialista, una participación ciudadana limitada y estrategias poco exitosas de la sociedad civil para incorporarse al diálogo público en los medios), se convierten, a su vez, en una agenda de investigación más amplia, pues estos fenómenos tendrán que ser descritos con mayor profundidad. ¿Qué condiciones hacen que los periodistas construyan las notas de la forma en que las hacen?, ¿cómo se vinculan los periodistas con los funcionarios públicos y los integrantes de la sociedad civil?, ¿qué estrategias emprenden los grupos organizados para incorporarse al diálogo público?, ¿qué elementos de radicalización se pueden observar en los discursos de estos diálogos?, ¿qué motiva a un lector a expresarse en la sección de correo de un periódico?, ¿qué imágenes se crean en la opinión pública de todos estos procesos? Estas son algunas de las preguntas que nos han asaltado luego de hacer el presente estudio y que tendrían que ser contestadas con la intención de seguir analiando los diálogos públicos.

Finalmente, este trabajo muestra que los periódicos tapatíos observados, como parte de una esfera pública en construcción, no consignaron encuentros fructíferos de diálogo entre ciudadanos y funcionarios públicos. Es evidente que desde el poder público no se impulsaron espacios de diálogo para construir colectivamente las políticas públicas de movilidad urbana del estado de Jalisco. A la postre, debido a presiones ciudadanas y políticas, la "Vía Exprés" no fue construida. Sin embargo, el abandono de este proyecto, lejos de construir un diálogo, significó un distanciamiento aún mayor entre el aparato gubernamental y la sociedad jalisciense. Un diálogo público de calidad habría implicado la construcción de un espacio plural, en el que se hiciera un esfuerzo por incluir a la mayor cantidad de actores sociales y políticos, y en el que se discutiera, colectivamente, el futuro de la movilidad urbana de la ciudad. 


\section{Bibliografía}

Acosta, R. (2012a). El diálogo y su calidad. Agenda de investigación sobre una herramienta social cotidiana. En R. Acosta (Coord.), El diálogo como objeto de estudio (pp. 9-26). Guadalajara. México: ITESO.

Acosta, R. (2012b). Calidad del diálogo público según una red de organizaciones ciudadanas de Jalisco. En R. Acosta (Coord.), El diálogo como objeto de estudio (pp. 359-378). Guadalajara, México: ITESO.

Ackerman, B. (1999). La política del diálogo liberal. Barcelona, España: Gedisa.

Avritzer, L. (2002). Democracy and the public sphere in Latin America. Princeton, EE.UU.: Princeton University Press.

Bautista, J. (2012). El diálogo en el diseño institucional y en la designación de los titulares de los organismos públicos autónomos de Jalisco. Propuestas para replantear su elección. En R. Acosta (Coord.), El diálogo como objeto de estudio (pp. 335-338). Guadalajara, México: ITESO.

Bobbio, N. (1997). Democracy and dictatorship. Cambridge, Inglaterra: Polity Press.

Cansino, C. (2000). La transición mexicana: 1977-2000. México: CEPCOM.

Carabes, G. (2010). Vía Exprés de Guadalajara, comentada por reales especialistas en el mundo [Video]. Recuperado el 7 de abril de 2012 de http://www.youtube.com/watch?v=0t9haAwK8Yg

Consejo Económico y Social de Jalisco-CESJAL. (Ed.). (2009). El Plan Estatal de Desarrollo Jalisco 2030. Una evaluación autónoma y ciudadana. Guadalajara, México: CESJAL.

Cunill, N. (1991). Participación ciudadana. Caracas, Venezuela: Centro Latinoamericano de Administración para el Desarrollo.

Dahlgren, P. (2000). Television and the public sphere: Citizenship, democracy and the media. Londres, Inglaterra: Sage.

Dennis, E. \& Snyder, R. (Coords.). (1998). Media and democracy. New Brunswick, Canadá: Transaction Publishers.

Emilio evita polémica por Vía Express. (4 de agosto de 2010). El Informador. 
Forester, J. (1999). The deliberative practitioner: Encouraging participatory planning processes. Cambridge, MA, EE.UU.: MIT Press.

Garcés, R. (2007). La construcción simbólica de la opinión pública. Estudios sobre el mensaje periodístico, 13 (pp. 55-81).

Garretón, M. \& Newman, E. (Comps.). (2003). Democracy in Latin America: (Re)constructing political society. Nueva York, EE.UU.: United Nations University Press.

Gobierno de Jalisco. (2010). Vía Exprés Gobierno de Jalisco [Video]. Recuperado el 7 de abril de 2012 de http://www.youtube.com/ watch?v= uHSAz56_8

González, I. \& Villar, R. (Comps.). (2003). Organizaciones de la sociedad civil e incidencia en políticas públicas. Buenos Aires, Argentina: Zorzal.

Graber, D. (2003). The media and democracy: Beyond myths and stereotypes. Annual Review of Political Science, 6, 139-160.

Gutmann, A. \& Thompson, D. (1996). Democracy and disagreement: Why moral conflict cannot be avoided in politics, and what should be done about it. Cambridge, MA, EE.UU.: Harvard University Press. Instituto Nacional de Estadística y Geografía-INEGI (2012). Censo de Población y Vivienda 2010. Recuperado el 7 de abril de 2012 de http://www.inegi.org.mx/est/contenidos/proyectos/ccpv/cpv2010/ Default.aspx

Habermas, J. (1989). The structural transformation of the public sphere. Cambridge, MA, EE.UU.: MIT Press.

Krippendorff, K. (1990). Metodología de análisis de contenido. Teoría y práctica. España: Paidós Comunicación.

Mendoza, M. (2010). Evita Emilio entrar en polémica por Vía Express. El Informador. Recuperado el 7 de abril de 2012 de http://www. informador.com.mx/jalisco/2010/223159/6/evita-emilio-entrar-enpolemica-por-via-express.thm

Morlino, L. (2005). Democracias y democratizaciones. México: Centro de Estudios de Política Comparada.

O’Donnell, G., Schmitter, P. \& Whitehead, L. (Comps.). (1994). Transiciones desde un gobierno autoritario: perspectivas comparadas. Barcelona, España: Paidós. 
Paoli, F. (2006). La transición incompleta. México: Granados Chapa Editores.

Ruiz, O. (2009, abril). Transformación de la esfera pública: Canal del Congreso y la opinión pública. En Documento de Trabajo del Centro de Estudios Sociales y de Opinión Pública, 67.

Sádaba, T. \& Rodríguez, J. (2007). La construcción de la agenda de los medios. El debate del estatuto en la prensa española. Ámbitos, 16, 187-211.

Thompson, J. (2011, enero/junio). Los límites cambiantes de la vida pública y privada. Comunicación y Sociedad, 15, 11-42.

Valdés, M. (2006). Radio y televisión en la transición mexicana. Treinta años de cambio político en México. México: Porrúa/UAM.

Whitehead, L. (2002). Democratization: Theory and experience. Oxford: Oxford University Press.

Zul, J. (14 de julio de 2010). La ex Vía Express. El Informador.

Fecha de recepción: 28/03/12. Aceptación: 06/06/12. 